\title{
Language Game Theory and Information Science: A Metatheoretical Investigation
}

\author{
Wang Lin \\ Department of Information Resource Management, School of Management, Tianjin Normal University (wanglinpku@163.com)
}

\begin{abstract}
This paper briefly introduces the language game theory put forward by late Wittgenstein, and then discusses the metatheoretical implications of language game for information science (IS) research. It demonstrates that compared with logical image theory, language game is more suitable and useful to the foundation of information science. It explores several aspects of IS in detail in the light of language game theory, including attaching the importance of conception of the document, reconsidering the traditional views and principles of information retrieval language and indexing practice, transforming the classical models in information retrieval to those based on late wittgenstein's views.
\end{abstract}

Key Words — Language game, Information science, Wittgenstein

\section{语言游戏论与信息科学：元理论视角下的研究}

\author{
王琳 \\ 天津师范大学管理学院信息资源管理学系, 天津 300387
}

\begin{abstract}
摘 要 本文简述了维特根斯坦晚期提出的语言游戏论, 并探讨了语言游戏论对信息科学研究的元理论意涵和价值。认为同逻辑图 像论相比, 语言游戏论更适合成为信息科学的理论基础, 作用也会更大。具体探讨了语言游戏论对信息科学若干研究领域的启示和价 值, 主要涉及信息观、知识组织及其工具检索语言、信息检索系统建设等方面。
\end{abstract}

关键词 语言游戏论, 信息科学, 维特根斯坦

\section{1. 语言游戏论简述}

语言游戏论（Theory of Language Game）是维特根 斯坦后期主要的哲学思想。与前维特根斯坦哲学的逻辑 图像论相对照, 维特根斯坦本人的哲学思想在其学术生 涯后期发生了重大的转变。在他的后期代表作《哲学研 究》中彻底抛弃了他前期哲学的根本性思想和概念, 对 包括他自己前期思想在内的逻辑语言主义进行了彻底批 判。维特根斯坦认为 ${ }^{[1,2,3]}$ : 逻辑图像论的语言观 (每一 个词都有一个意义, 意义是词代表的对象; 命题是描述 事实的逻辑图像, 只有可判断真伪的命题才有意义等） 不能解释所有语言现象; 人类所使用的所有语言实际上 不过是如同儿童玩要或人们常玩的各种游戏, 可以把由 语言和语言交织在一起的行动所组成的整体叫做“语言 游戏”; 采用此概念的意图是想强调语言和日常生活的联

本文为国家社会科学基金青年项目“中小企业经理人的信息搜寻 影响因素与行为模式研究” (项目号:13CTQ040)资助的研究成果。
系, 以及生活中语言运用的多样性。直接指称定义 (ostensive definition) 只是语言游戏的一种; 有真伪而 言的语言游戏也只是众多可能游戏中的一种; 语言游戏 论认为语言是没有本质结构的, 词的意义就是它在语言 中的用法, “意义即使用”，使用是工具性的; 语言的任 何使用都在特定的生活实践中进行, 要了解一个命题的 意义, 就看它在何种环境下被使用; 遵守规则是语言游 戏的基础, 任何一种语言游戏的规则都根植于生活形式 之中, 都在语言实践中建立起来, 因此遵守规则可以说 是一种习惯、一种制度或一种实践, 语言游戏继而归根 到底是一种人类文化现象。哲学所应做的是考察不同的 具体语言游戏, 从中掌握语言的不同用法, 根据句子和 词语的使用来确定它们的意义。

\section{2. 语言游戏论作为信息科学元理论的意涵与价值 与前期维特根斯坦哲学的逻辑图像论相比, 后期维 特根斯坦的语言游戏论作为元理论对信息科学理论建设}


的影响要小的多。信息科学核心领域如信息检索、知识 组织的许多理论成果都是建立在逻辑图像论的元理论假 设上的。正如文献[4]所指出的那样: “许多信息科学家 对维特根斯坦后期走向其早先思想的对立面而感到遗 憾, 因为逻辑图像论看起来比语言游戏论要更适合于形 式化的信息检索。”但这并非是说语言游戏论对于信息科 学是一种不合时宜的元理论, 而是我们迄今仍没有全面 地认识到它的理论潜力。深入地研究语言游戏这一元理 论很可能会促使信息科学理论在很多方面产生根本性的 变革和理论品质的提升。实际上这种情况在下述的信息 观、知识组织及其工具检索语言、信息检索系统建设等 方面已经初现端倪。应指出的是, 由于语言游戏论本身 比较深奥 (陈嘉映先生就曾说过《哲学研究》不是一本 容易读懂的书), 信息科学界对它的理解和应用仍有待深 入, 目前的研究只是初步的。

信息观中关于“信息是什么”的争论已经由来已久。 弗莱赫曼则在引述了南波格质疑“信息是什么”这个问题 的问题本身来源和合理性之后, 分析了语言游戏论对于 信息观的作用 ${ }^{[5]}$ : 维特根斯坦把语言游戏归结到实践的 范畴, 对于语言实践的关注削弱了意义的哲学理念。由 于信息性（informativeness）遵从意义性, 因而语言游戏 对实践的关注也削弱了把信息视作有意向的实体这一思 想的重要性; 维特根斯坦所举语言游戏的很多例子都涉 及了文献的实践 (documentary practice), 正如他对奥古 斯丁图画 (Augustinian picture) (与信息图景观存在密切 联系) 的批评使关注的焦点从意义的心智图像转向了语 言的实践一样, 这也使我们信息科学的注意力从信息的 心智图景转向到文献的实践。维特根斯坦语言游戏的哲 学视角意指要从信息理论变迁到对文献实践的描述, 而 文献实践的属性主要有物质性、制度性、社会规范性和 历史性, 这些属性为以文献实践观为出发点的信息哲学 研究提供了有益的开端; 信息, 即使是科学信息, 其产 生也依赖于特殊文化的、由历史决定的、具有制度规范 的文献实践, 对文献实践的关注能揭示特定的文献如何 在特定的时空和特定的社会文化领域中才变得具有信息 性。可见, 这样的观点即是认为文献的信息性依赖于与 之相关的特定实践，文献实践是信息本体基础。

无独有偶, 我国学者在上世纪九十年代也曾提出过 “文献信息学”的理念, 认为在强调信息这一当时的学术 “热潮”时, 也应当突出文献的概念而不是舍弃它, 故而 合称“文献信息”, 文献信息学则是能覆盖图书馆学、信 息科学和档案学的综合性学科 ${ }^{[6]}$ 。这种对学科的理性思 考与西方以语言游戏论为元理论基础的文献实践观不无 “暗合”之处, 两者都把文献理论和文献工作的研究摆在
了显著的位置。在我们今天探讨信息科学的学科融合和 创新时, 有必要回到信息科学学科发展的起点——奥特 莱创立的文献学那里去深入思考问题。如果说信息科学 要巩固和发展学科人文传统的话; 那么信息科学就要首 先强调自身悠久的“文献传统”, 因为只有这样才能使学 科在自身内部产生人文传统的历史底蕴。信息科学的“文 献传统” 与知识、意义、学习、描述、语言和模糊性相关 联, 它根植于文化研究、人文和质化的社会科学之中[7]。 而语言游戏元理论认识到语言游戏是一种文化现象, 文 献实践依赖于社会文化、历史和制度规范, 这些无疑会 为新时期信息科学理论的“文献传统”提供丰富的思想源 泉, 并成为其重要的元理论基础。由此也可看出, 文献 的概念在信息科学中仍有不可轻视的作用, 研究在重视 知识概念的同时, 不能唯此独尊, 而是要辩证地看待知 识与文献、信息的关系。

在知识组织和知识组织工具的研究中, 基于语言游 戏元理论的观点与传统观点也有所不同。

首先是与传统分类和主题观的对立 [8]: 传统分类法 是与知识的客观主义理论相关联的, 其基础是逻辑的属 种关系, 事物的本质是分类的标准, 而维特根斯坦则认 为如同一个家族的人们之间不存在每人都具有的共有特 征一样, 归属于同一类目下的事物也不必然地存在共有 特征。当词语的意义不是语言行为的功能时, 它的实例 不需要有共同的本质属性, 因此根据维特根斯坦这一观 点, 我们要用家族相似而不是相同属性的匹配来比喻类 目的形成; 传统的主题观是建立在语法模型基础上的, 属于意义理论中的实证主义方法和还原主义。而实际上 主题是复杂的, 在语言学上有时是非确定性的, 它所表 述的是一组概念体系, 用于表示主题的词语意义的可变 部分只有在概念体系中被加以情境化时才能呈现, 用于 联结主题的词语间关系更有可能通过研究用户网络查寻 和导航中的语言行为来发现。这些正是语言游戏中“意义 在于使用”和使用的生活实践观的具体体现。

其次, 在标引上, 与心智主义的图式不同 ${ }^{[9]}$ : 心智 主义图式认为标引员的心智活动是由他内心隐秘的一些 规则来指引的, 文本标引行为被解释为头脑中心智规则 的唤起, 这些规则能自发地从对文本的心智表示中产生 出标引的心智表示, 理论所要探究的就是要发现这些规 则的精确形式。然而, 根据语言游戏论, 我们对规则的 把握并不在于将其与图像、心智表示和解释相联系, 规 则是在我们的行动中所展示的, 因此标引规则最好应当 被视为特定社会实践的工具。理论所探究的不应是发现 人潜意识遵循的规则, 而是以明确的目的去主动构造外 显的、表述清晰的和严格的标引规则。而要构建标引规 
则, 就要理解生成规则的社会情境和规则实践的社会角 色 (包括规则嵌入社会的方式、主旨、目的等), 探究领 域中规则产生的历史、经济、政治等情境。构造标引规 则是为了使特定的文本检索更为便利, 而文本检索标示 着一组特定社会实践集合, 为此构造标引规则就要依赖 于对历史性的、真实社会世界中检索实践的通透理解。

再者, 知识组织的工具一一检索语言是有多种多样 的类型的, 使用每一种检索语言来组织知识都是一种特 定的语言游戏 [10], 正如一种语言游戏不能代替或化约 为另一种语言游戏一样, 各种不同检索语言也都有其独 特的功能和作用, 彼此间是不能完全替代的。设计出一 种统一的逻辑完美的、终极清楚的综合性检索语言不过 是弗雷格和罗素所设想的语言在信息科学中的表现, 是 殊难企及的。

在信息检索系统研究中, 最为流行的是信息的逻辑/ 数据模型, 这是缘于计算机在本质上是逻辑机器的缘故。 依此模型设计出的系统在处理和检索精确的、高度确定 性的内容如姓名、地址等时得心应手, 但对于确定性程 度低的“智力内容”(intellectual content)的检索就力不从 心了; 逻辑/数据模型要求意义的绝对确定性, 其对于语 言的基本假设是早期维特根斯坦的思想, 即认为存在掌 控语言使用的基本逻辑语言。依据语言游戏论的观点, 将逻辑/数据模型试图应用到语言和意义的所有方面显 然是不会成功的, 尤其是在对确定性低的信息或智力内 容的检索上[11]。

\section{3. 结论}

语言游戏论是建构主义的重要理论流派之一, 亦是 倍受后现代主义者推崇的哲学理论。在对语言游戏论做 了简要介绍之后, 本文从元理论层次阐述了语言游戏论 对信息科学的作用与价值, 涉及的方面主要包括在学科 中重新强调文献概念和文献实践的重要性, 对知识组织 和知识组织工具的传统观点的重新思考, 信息检索中逻 辑模型的局限性和开发新模型的必要性等方面。当前语
言游戏论在信息科学中的引进和应用都处在初级阶段, 但可以预见, 随着学界对基础理论研究重视程度的提高, 以及由信息工作实践中不断暴露出的新问题所引发的重 构学科底层理论需求程度的加大, 语言游戏论在信息科 学元理论中会占据越来越重要的地位, 其作用和价值会 进一步地凸显。

\section{参考文献(References)}

[1] Huang, G.G. The logic of social science. Beijing: Press of China Renmin University 2006

[2] Liu, F.T. Mordern Philosophy in Western Countries. Beijing: People Press, 2000

[3] Chen, J.Y. Language Philosophy, Beijing: Press of Peking University 2003

[4] Hjørland, B. Theory and metatheory of information science Journal of Documentation. 1998 54(5): 606- 621

[5] Frohmann, B. Documentation Redux: Prolegomenon to (another) philosophy of information. Library Trends 2004 52(3): 387-407

[6] Zhu, J.L. Introduction to Documentary Informatics Beijing: Bibliography Press 1992

[7] Buckland, M. The landscape of information science: the American society for information science at 62 available at: http://www.ischool.berkeley.edu/buckland/asis62.html

[8] Svenonius, E. The epistemological foundations of knowledge representation Library Trends. 2004.52(3): 571-58

[9] Frohmann, B. Rules of indexing: a critique of mentalism in information retrieval theory. Journal of Documentation 1990 46(2): 81-101

[10] Svenonius, E. The intellectual foundation of information organization Cambridge MA: MIT Press 2000:1-14

[11] Blair, D. Wittgenstein, language and information: "Back to the rough ground!” In Crestani, F \& Ruthven, I (Eds) Proceedings of CoLIS5 Springer 2005:1-3 